\title{
Model Based Solver Application (MBSA) For Manufacturing Industries
}

\author{
Nagappan Annamalai ${ }^{1}$, Shahrul Kamaruddin ${ }^{2}$, and TS Yeoh ${ }^{3}$
}

\begin{abstract}
Problem solving is a fundamental element in retaining and achieving operational excellence of a manufacturing industry. There are numerous problem solving models are being used widely but the problem is to determine an optimal or a poka-yoke solution according to the constraints of respective manufacturing industry. Nevertheless, optimal solution for problems has become a great challenge in view of the fact that existing problem solving model is highly vague where at times it is being neglected either due to ineffective or too complex. This leads to individual getting confused and not able to devote their time successfully on any of the problem solving models. The most common approach of problem solving model is via trial-error or insight pattern where problems are being tackled and resolved in a quick manner, which it eventually contains the problem, instead of identifying the actual root cause and resolve the problem for good. The paper will discuss on a newly developed model based solver application, which is capable to determine the optimal solution in industries mainly to avoid any repeated equipment breakdown occurrences, quality issues, and high spare maintenance spending.
\end{abstract}

Keywords - problem solving, manufacturing, problem definition, optimal solution.

\section{INTRODUCTION}

The problem solving model (PSM) is an important tool for solving a problem. The key element is to understand and define the problem statement clearly to achieve the ultimate goal. The theoretical basis for the present study is the research on problem-solving derived from the work of Newell and Simon [1], which conceptualized the process that people employ to solve problems. In this conceptualization, problems are concepts that occur in a problem space. The problem space contains three elements: a problem state, which is the information that the problem solver must know about the problem; a goal state, which constitutes the solution to the problem; and a search space, which consists of all the strategies that may be employed to solve the problem.

Nagappan Annamalai ${ }^{1}$ is with the Intel Technology and studying at Universiti Sains Malaysia, Penang. Malaysia (60124501251;

Dr. Shahrul Kamaruddin ${ }^{2}$, was with Universiti Sains Malaysia, Penang. Malaysia. He is now with Universiti Teknologi Petronas, Perak. Malaysia

Dr. TS Yeoh ${ }^{3}$ is with Inno Planet, Penang. Malaysia

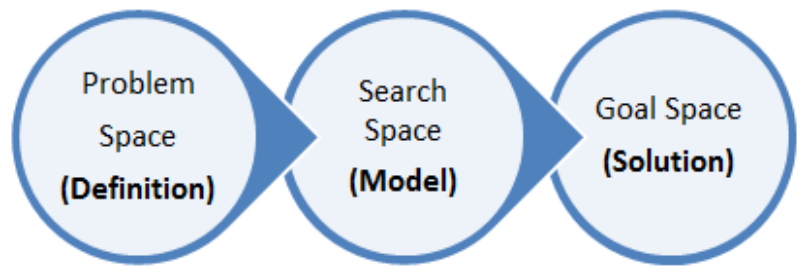

Figure 1: Three space in PSM

The PSM is a key element in real-world processes, such as in manufacturing industries. In manufacturing industries, operational excellence must be achieved by maintaining equipment sustainability and stability. Operational excellence is an element of organizational leadership that stresses the application of a variety of systems and tools toward the sustainable improvement of key performance indicators. Therefore, a precise PSM is important in quickly determining the root cause of a problem and fixing the issue. The manufacturing industry is also an environment that utilizes the design of experiment of PSM application to achieve operational excellence. Manufacturing firms apply various models to perform problem solving based on problem complexity. In a manufacturing environment, problem solving requires not only skilled individuals who are technical experts but also the ability to understand the PSM for tackling complex problems. Problems typically begin as small "baseline issues," become acute and in some instances chronic, and finally transform into big or complex issues. For highly complex problems, the solution analysis requires discrete details because a combination of issues may be involved. This condition is true for the industry of interest in this research, which requires an easy and structured PSM. In a study of organizational decision making [2], it is concluded that one half of problem-solving studies fail because technical experts are hasty and tend to cut corners when implementing the important steps of the PSM, including problem definition, diagnosis, setting of objectives, identification of alternatives, and implementation.

The manufacturing industry uses numerous models to achieve operational excellence. The most popular models are the following: seven-step problem solving (SPS), theory of inventive problem solving (TRIZ), analytic hierarchy process (AHP), and Kepner-Tregoe (KT) model. These models are usually neglected because of either ineffectiveness or complexity. Hence, experts become confused and fail to focus 
on any of the approaches. At times, experts are puzzled by the containment and control solution paths. In many instances, problems are quickly tackled and resolved, in which case the issues become contained, instead of identifying their root cause and resolving the issue/problem for good. Experts also tend to become unfocused while working on a problem because of unclear problem statements and lack of analyses. It was mined and conducted a study aimed at understanding the problem statement and analyses prior to the problem identification model [3]. The key to forming a clear picture of an issue is understand the connection between the entity and the value. This is where a new PSM known as Model Based Solver Application (MBSA) has been developed and discussed.

MBSA is separated into 4 stages starting from problem definition, which segmentize and characterize the problem statement into wider scope. Meanwhile, modeling the defined problem is proposed in second stage of MBSA to analyze and validate the findings for optimal solution in third stage. Inventive solution via engineering contradiction in the third stage of MBSA provides a narrowed and optimal solution for the problem. Finally stage 4 , which proliferates the inventive solution which ensures a proper implementation across the affected problem industries. The robustness of the MBSA model has been verified and validated through different case studies in a manufacturing industry. The results obtained from each of the case studies had proven the robustness of the MBSA in determining the optimal and inventive solution. Overall MBSA model has proven its capability in providing systematic and effective way of problem solving under different circumstances.

\section{LITERATURE REVIEW}

Most problem-solving research concentrates on simple or well-defined problems [4]. These problems can be described clearly in terms of their nature, the elements that constitute an acceptable solution, and the various strategies that can be used to achieve an acceptable solution. However, complex and ill-defined problems are often complex because defining each element of the problem space is difficult [5].

The fundamentals of problem solving are critical, and even seasoned industry experts can get lost in all the models [6]. A data source (Figure 2) explains why the search for root causes can be slow. The reasons include a wrong problem statement, a considerably difficult problem, conclusions without data support, misinterpreted data, assumption of only one root cause, and unclear expectation.

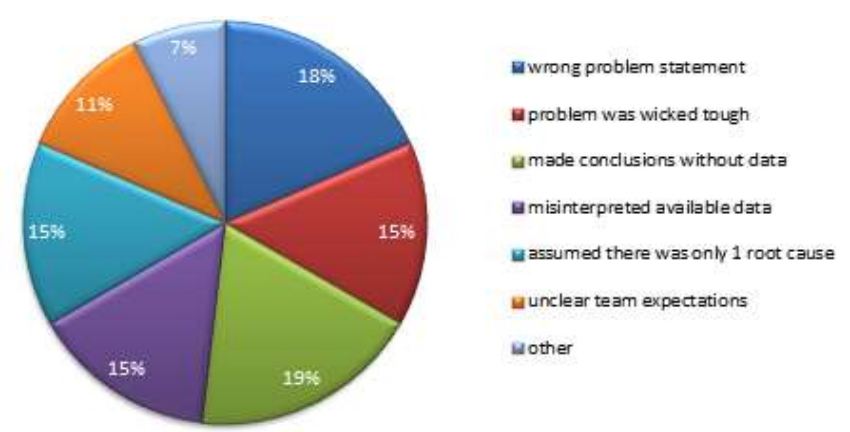

Figure 2: Pie Chart Of Weak Root Cause Analysis

PSMs are divided into four key elements, namely, algorithm, heuristics, trial and error, and insight [7].

\section{A. Algorithm}

An algorithm is a step-by-step procedure that will always produce a correct solution. A mathematical formula is a good example of a problem-solving algorithm. Although an algorithm guarantees an accurate answer, it is not always the best approach to problem solving. As algorithms are time consuming, they are not practical for many situations. For example, determining all possible number combinations of a lock using an algorithm would take a long time because of the various patterns that exist (Figure 3).

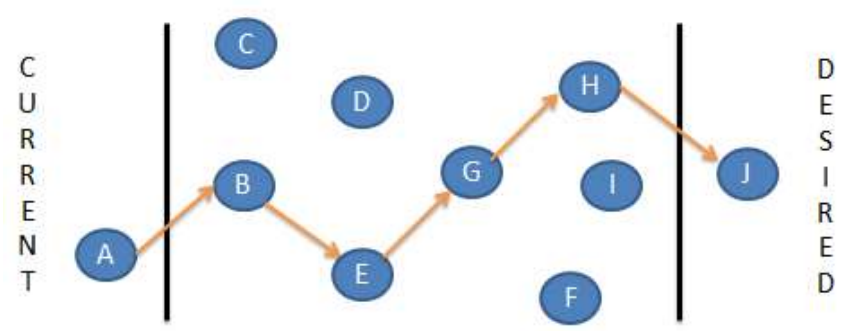

Figure 3: Algorithm Pattern

People employ two types of models to navigate the problem space when solving problems. The first model involves algorithms, which are defined as strategies that guarantee solutions. Algorithms tend to be useful for problems in which clearly identifying all the features of the problem space is possible. The second type of strategy is called heuristics, which are strategies that improve the chances of solving a problem but cannot guarantee a solution.

\section{B. Heuristic}

Heuristics refer to experience-based models for problem solving. A heuristic is a mental rule-of-thumb strategy that may or may not work in certain situations. Unlike algorithms, heuristics do not always guarantee a correct solution. However, using this PSM allow people to simplify complex problems and reduce the total number of possible solutions to a manageable set called a heuristic pattern [8], as illustrated in Figure 4. 
Proc. of the Third Intl. Conf. on Advances in Mechanical, Aeronautical and Production Techniques - MAPT 2015 Copyright (C) Institute of Research Engineers and Doctors, USA .All rights reserved. ISBN: 978-1-63248-059-0 doi: 10.15224/ 978-1-63248-059-0-69

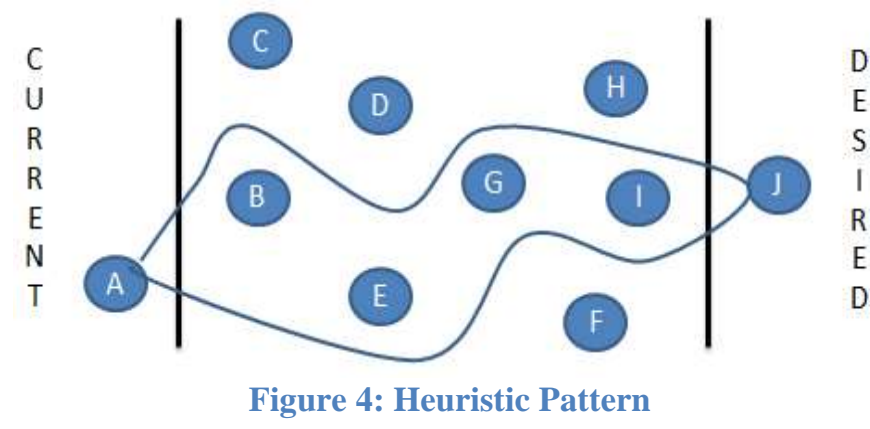

Problems are often complex because identifying their characteristics is not possible. Complex problems are often solved using heuristics [1]. A number of heuristics have been identified in the literature. The two general types are forward reasoning and backward reasoning [9]. Forward reasoning models involve the problem solver making a smooth traversal from problem state to goal state. Forward reasoning is often used by people who are knowledgeable in the topic or domain of a problem and capable of choosing each step without checking on the progress of the problem-solving process. Backward reasoning involves trying models and monitoring whether they have moved the problem close to the goal. Heuristics are particularly useful in solving complex problems. Heuristics involve discovery, problem solving through experiments, and trial and error methods.

\section{Trial And Error}

Trial and error is a fundamental PSM. A trial-anderror approach to problem solving involves trying a number of different solutions and ruling out those that do not work [10]. This approach can be a good option when a limited number of options are available. With many different choices, the possible options should be narrowed down using another problem-solving technique before trial and error is attempted.

Trial and error is a common backward reasoning strategy employed by people who do not have extensive experience or knowledge in a problem domain. With problems that require new and creative solutions, a strategy called problem finding [11] has been identified as an important ingredient of successful problem solving. Problem finding is defined as exploring the problem extensively before attempting a solution while being prepared to change directions when necessary during problem solving [12]. Figure 5 depicts an example of a trial-and-error pattern.

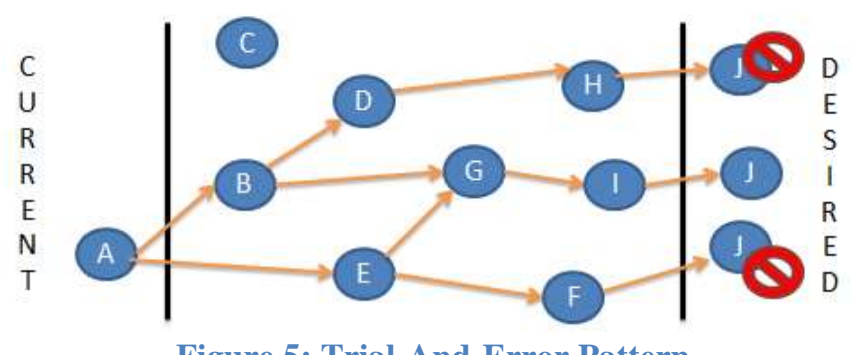

Figure 5: Trial-And-Error Pattern

The trial-and-error approach is successful when applied to basic problems. Trial and error is generally an attempt to find a non-optimal solution in cases in which little knowledge of the subject is required.

\section{Insight}

Insight is the understanding of a specific cause-andeffect chain (CEC) in a specific context. In other words, insight is an understanding of the CEC based on the identification of relationships and behaviors within a model. An insight manifests itself suddenly, such as when understanding how to solve a difficult problem [13]. Insight can occur because you realize that the problem is actually similar to something that you have dealt with in the past; however, the underlying mental processes that lead to insight occur outside awareness in most cases [14]. PSM optimization involves searching for the best solution among a set of feasible solutions [15] based on a given criteria (refer Figure 6). Optimization between algorithms, heuristics, trial and error, and insight can present a simplified and structured model for problem solving.

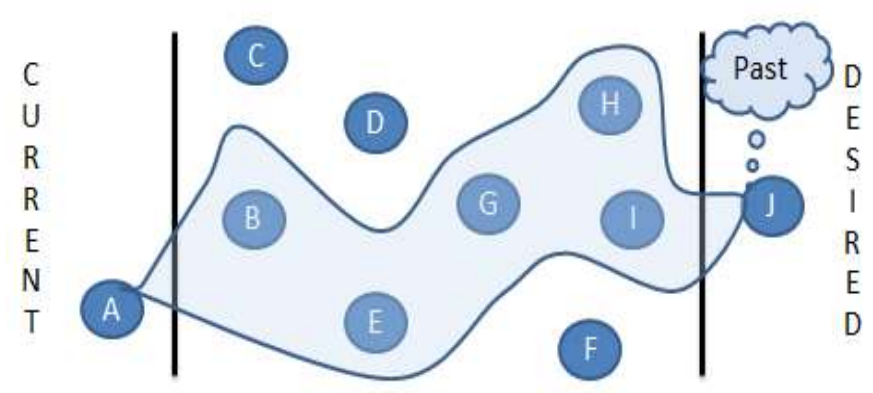

Figure 6: Insight Pattern

The previous section explained four key elements of a problem-solving strategy. These elements have led to the convergence and optimization of a new research PSM. The following section discusses widely used PSM and the incorporation of the four elements in these models. The focus of the discussion lies on the methods, application, manufacturing industry, AHP, FMEA, KT, TRIZ, 8D, SPS, EEC, engineering, and process. This chapter also cites the methods that have been used in manufacturing industries, such as automobile, food and chemical processing, and other fields that are related to manufacturing.

\section{FINDINGS}

Most innovations for problem solving in manufacturing industries involve the elements of trial and error and insight. However, these elements are highly dependent on luck and thus fail to consider all possible solutions. Algorithmic and heuristic paths are systemic processes that reveal the exact or closest total solution space. These paths can quickly converge into an optimal solution through systemic analysis. They can also provide a comprehensive coverage of the solution space and in turn enable a successful selection of a PSM. Table 1 tabulates the mapping of individual PSMs to the model elements. 
Proc. of the Third Intl. Conf. on Advances in Mechanical, Aeronautical and Production Techniques - MAPT 2015 Copyright (c) Institute of Research Engineers and Doctors, USA .All rights reserved. ISBN: 978-1-63248-059-0 doi: 10.15224/ 978-1-63248-059-0-69

Table 1: PSM Mapping To Model Element

\begin{tabular}{|c|c|}
\hline Elements & Problem Solving Model \\
\hline Trial-Error/Insight & Ishikawa Fishbone \\
\hline Trial-Error/Insight & 8-Discpline (8D) \\
\hline Algorithm & TRIZ \\
\hline Heuristic & FMEA \\
\hline Algorithm & 7-SPS \\
\hline Algorithm/Insight & AHP \\
\hline Trial-Error/Insight & KT \\
\hline
\end{tabular}

Successful problem solving is clearly guided by a model, is unconstrained, is associated with controlled urgency, involves adequate resources, and utilizes an available framework for sharing. Meanwhile, unsuccessful problem solving has limited model guidance, is significantly constrained, is chaotic or inertial, involves inadequate resources, and indicates limited understanding. Such problem solving is not systematic and fails to unify the cognitive perspectives of stakeholders. They also fail to promote positive system changes or deliver closures that satisfy industry requirements. The present study proposes a new concept that integrates these findings into an elaborate PSM. The next section discusses the evolution of elements and PSMs in the S-curve trends.

Technologies, products, models, and patterns tend to evolve slowly along an S-curve during the early stages [16]. This slow evolution is followed by a rapid growth to maturity, after which the development slows down and declines in the late stages. This sequence of events can analytically describe the S-curve through the following logical equation:

$$
P(t)=\frac{K P \circ e^{r t}}{K+P \circ\left(e^{r t}-1\right)}
$$

where $r$ is the parameter that characterizes the growth rate, $\mathrm{K}$ is the capacitance of the medium, $P$ o is the initial number of media, and $P$ is the number of media at time $t$.

This trend evolution has been observed over time across a wide range of technologies and products. Understanding the location of a particular pattern on this S-curve continuum can have important implications for strategic development and innovation investment. This S-curve includes the understanding and positioning of a technology or pattern that will lead to answers to the following questions:

- Where are we today on the S-curve?

- What is the development potential under the current action?

- When should we consider jumping to the next S-curve?

The S-curve has evolved into a nonlinear dynamical system in which each trend depends on its previous trends [17]

$$
\begin{aligned}
& G n=f(t ; G n-1 ; E n-1), E n-1=f(G n-1) \\
& \text { where } \\
& \text { G - Generation } \\
& t \text { - Time } \\
& \text { E - Environment } \\
& n \text { - Generation Number }
\end{aligned}
$$

As shown in Figure 7, the problem-solving pattern has evolved from an algorithm that requires a step-by-step process to a time-consuming model. This evolution has created a mental block for users, especially with algorithm-based models requiring a tedious process. A heuristic pattern has since taken over, and most problem-solving approaches have been simplified with past experience and knowledge. This evolution does not actually fulfill engineering requirements because it does not offer exposure to those who lack experience and problem definition is not deep enough to achieve progress. The trial-and-error pattern has also emerged, providing a platform in which idea generation is a backward proliferation activity based on experiment design. This pattern may not fully control a problem because it leans toward the rapid generation of a solution without a detailed understanding of the problem statement. The insight pattern is another product of the evolution of problem-solving patterns. This pattern is based on past experience, making it similar to the heuristic approach and represents a convergence with the trialand-error pattern. These four cycles show that in their quest to achieve a simplified PSM, experts neglect the root cause analysis because they prioritize a rapid search for solutions. The latest trend shows the emergence of a clustered problemsolving pattern. Each existing PSM has strengths and weaknesses, but they are generally focused on strengthening the problem definition space and employing a rapid problemsolving approach. A prospective next step is the development of a PSM that clearly addresses the deficiencies in the problem definition space through the introduction of inventive analysis, model space, and solution space. In the process of developing this PSM, contradictions must be fully understood and mitigated to achieve foolproof solutions.

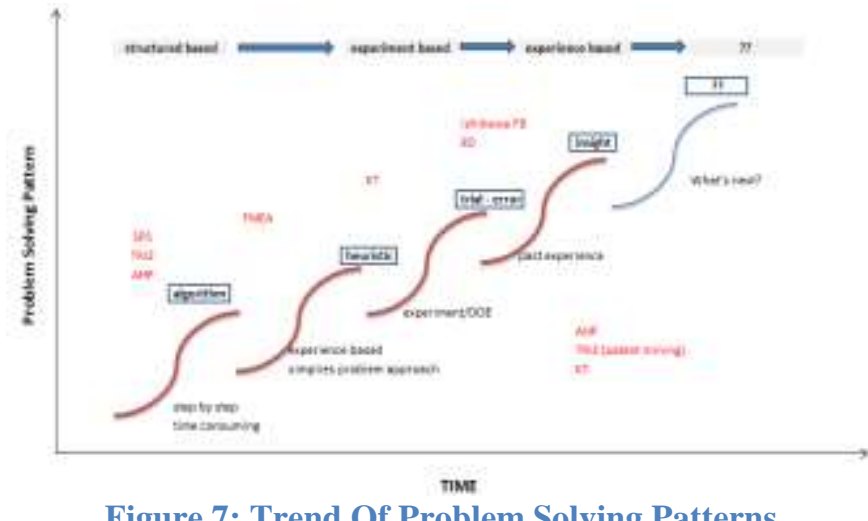

Figure 7: Trend Of Problem Solving Patterns

The literature review shows a lack of related work on problem identification, with details being mainly focused on manufacturing industries. This issue presents another key area 
of improvement. When an appropriate and systematic PSM is not available, teams are bound to spend a significant amount of time in analyzing problems instead of focusing on solving the actual root cause of issues. An innovative analysis approach based on existing models has recently emerged. This trend calls for the initiation of changes to develop the new PSM. The new model is expected to focus on the problem definition and model development to systematically identify the actual root causes of problems as well as potential solutions. Furthermore, the new PSM requires validation through several industry case studies to ensure its effectiveness

\section{CASE Study Research}

The research aim involves "how" and "why" questions and that the use of case studies should be the preferred research strategy [18]. At least six kinds of case studies are based on $2 \times 3$ matrixes; single or multiple case studies are exploratory and descriptive. The multiple case studies approach is chosen because it can access industry problem-solving practices in the real world. This research can be categorized as a descriptive case study based on quantifiable evidence that explains the occurrence of an event. During the theory/model development process, logic replaces data as the basis of evolution [19]. In this case, the "whys" that underlie the reconstituted "whats" and "hows" must be explained. Case study research is suitable for simulating the developed model and justifies the workability of the theory. An unstructured theory involves a few constructs and related propositions with some empirical or analytical grounding, but weak conceptualizations and underlying theoretical logic limit this theory. No example in the PSM literature is based on a case study approach, with most examples rooted in the generic problem-solving approach and mathematical-algorithm solving.

Research findings are obtained through in-depth analyses of cases [20]. There is method of when and how to use case studies in model development [21]. Supporters of case study research are concerned with understanding the depth ("why"), whereas rationalists tend to focus on explanations and predictions ("what" and "how"). These methods can be clustered to derive an enhanced model. Problem situations are mostly from practices or case studies. A case study method is a focal point in recognizing hidden problems. The problem statement or problem recognition is a baseline in solving the correct problem.

The case study method is a specific field research method. Field studies investigate problems as they occur without any significant intervention of the researchers. A case study refers to a detailed analysis of an individual case [22], assuming that "one can properly acquire knowledge of the problem from intensive exploration of a case study."

As elaborated in the literature review, the PSM is intrinsically difficult to use when resolving chronic or complex problems. One of the key inputs to problem solving is using an MBSA, which can allow engineers to easily rectify issues with full-blown or permanent fixes. If the model is accurate and user friendly across a wide range of scenarios, then the fixes to chronic problems will be accurate. Otherwise, using this model can lead to wrong decisions or solutions that can sometimes be expensive, result in continued high yield losses, and weaken equipment behavior. These potential effects justify the need to develop, design, and apply the MBSA methodology, which can accurately identify the root cause and fix the problems to ensure that factories operate smoothly and without any complex or unfixed issues (walking wounded).

A highly accurate MBSA can augment equipment stability by addressing the limitation of current problemsolving methods. For complex or chronic problems that cannot be solved or fixed permanently, the response from the MBSA can be used, as depicted via case studies. The MBSA can demonstrate a powerful result for real industry issues with accurate fixes.

Industrial case studies are discussed and validated in this research. The case study selection is based mainly on the key critical issues that occur in specific semiconductor manufacturing firms over the years. The MBSA approach is used to validate the robustness of the problem model.

\section{MBSA}

The head of research for General Motors [23], says, "A problem well-stated is a problem half-solved." In the literature review, we discussed fact finding and described the distance between the problem statement and search for solutions. This distance is expected to be addressed through the MBSA (Figure 8). Stage 1 is problem definition, which is a critical stage in PSMs. If the problem statement and analysis are stated wrongly and are inaccurate, then the following stages will not lead to the actual root cause and solution. Stage 2 is focused on modeling and emphasizes model validation based on the findings from the problem analysis. Stage 3 complements the previous stages by dealing with the solution identification. Stage 4 focuses mainly on the implementation of the solution, which is expected to show significant results by solving the problem. The MBSA looks into the existing PSM distance and identifies the opportunity to improve its use while exploring the actual root cause.

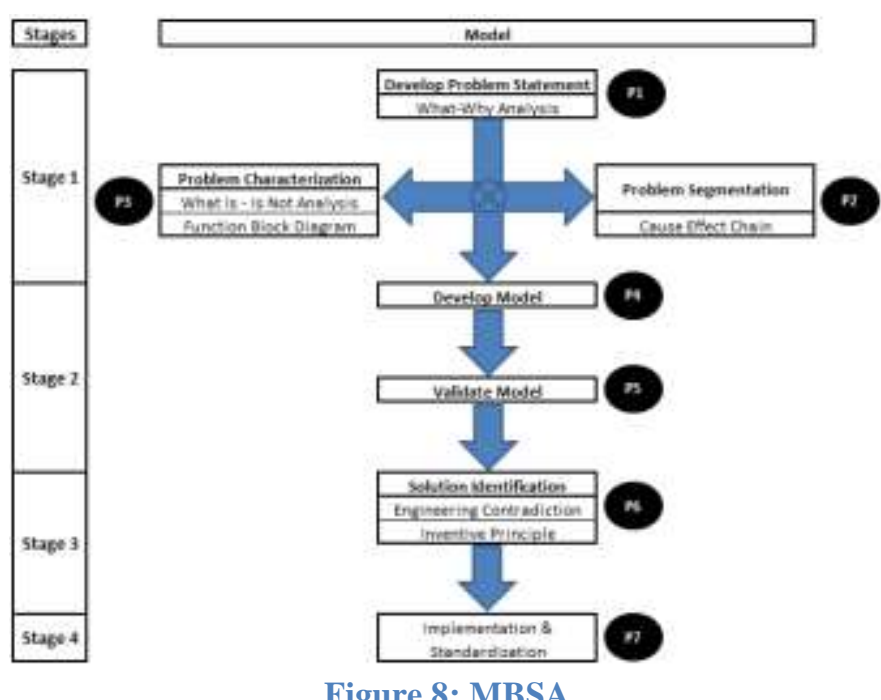

Figure 8: MBSA 
In many instances, this stage is neglected because Stages 1 to 3 are not well discussed, leading to the recurrence of the problem. If a solution is not foolproof, then the management would disregard the implementation stage, in which investment costs are incurred. In implementation, the solution is proved essential, and additional data points are required along with the factors that influence this stage [24].

The novelty of the MBSA is the problem definition model stage, in which in-depth analysis involves three key phases: problem statement, segmentation, and characterization (problem analysis). These phases ensure that no distance exists between the problem definition and the solution identification. Figure 9 shows a simple analysis of the PSM.

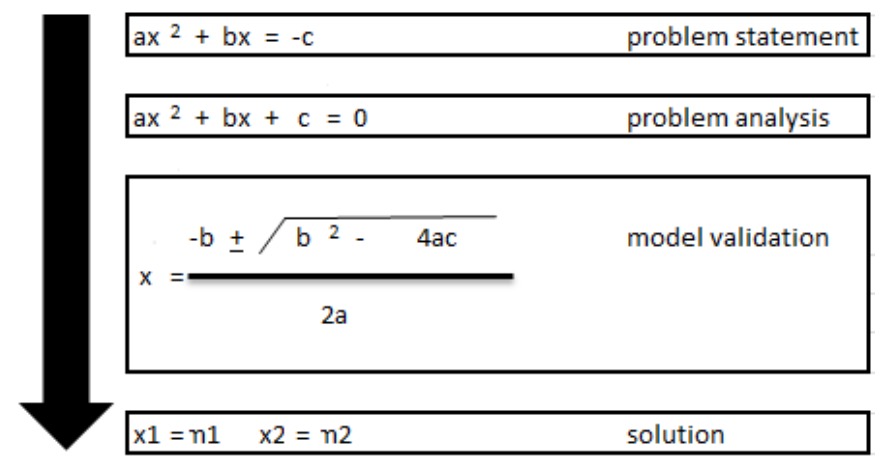

Figure 9: Analysis Of The PSM

In the present work, the difficulty lies in the derivation and clustering of the three problem analysis phases so as to ensure that a robust problem identification model is generated. Various types of problems are presented in the research of MBSA. This model consistently demonstrates rapid identification of actual root causes and solutions for complex manufacturing problems.

\section{CASE Study}

Class test is one of the module types under the test process. This test consists of three submodules, namely, tester, handler, and test interface unit (TIU) (Figure 10). This case study focuses on the TIU module, which is an interface hardware that test the product capability.

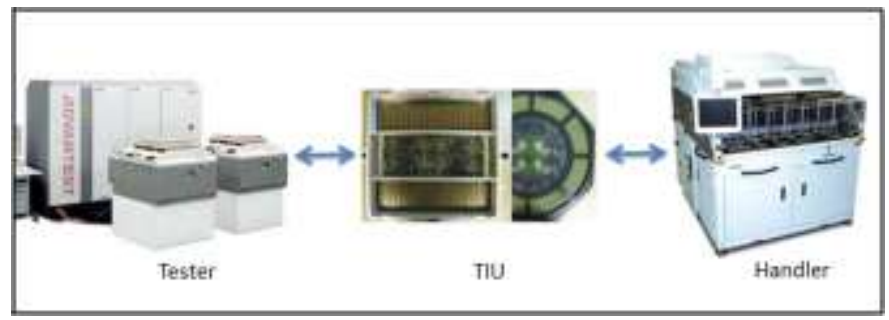

Figure 10: Class Test Module

The problem statement is a specific bin failure that induces high yield loss. This failure leads to unnecessary equipment downtime that affects productivity. The cost of the exploration work is at the crux of the problem. Through the FMEA, we find no effective solution for controlling the specific B13xx failure, with the containment plan being swapping or changing the hardware when an alarm is triggered.

In Stage 1, the problem statement is reviewed to achieve a clear picture of the issue. A what-why analysis (Figure 11) is then performed to understand the importance of solving the problem and the obstacles that hinder problem resolution.

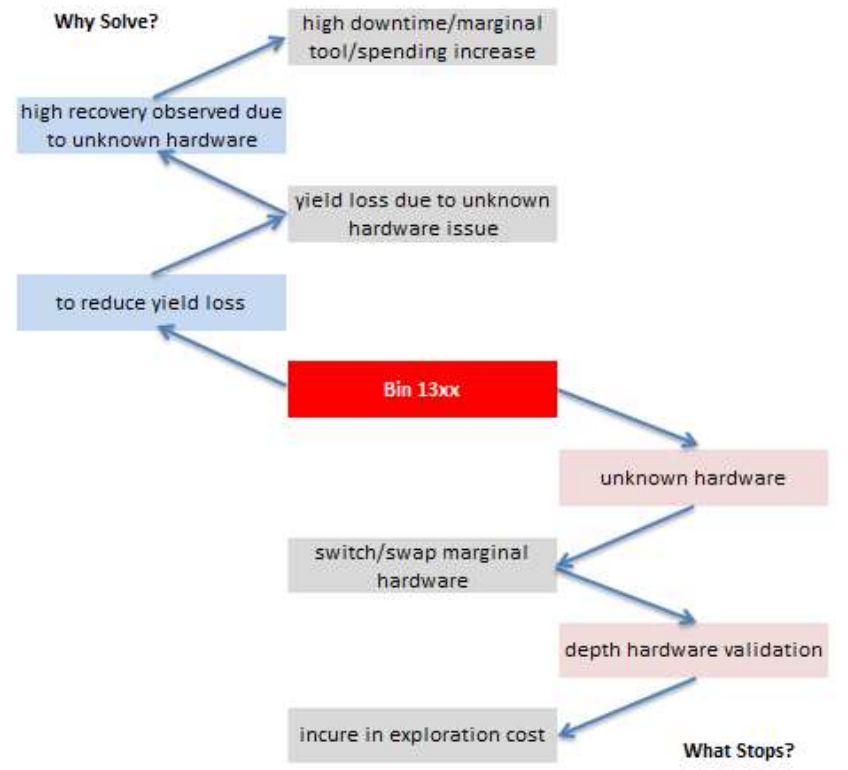

Figure 11: What-Why Analysis (B13xx)

The next step is problem segmentation analysis through the CEC, which is aimed at understanding the whys and identifying the potential key disadvantage of the high yield loss issue of the B13xx. Figure 12 shows the related CEC analysis.

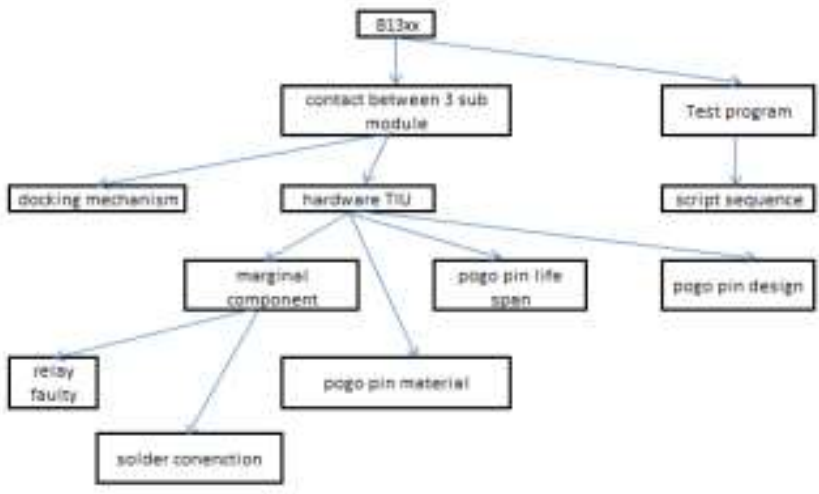

Figure 12: CEC Analysis Of High Yield Loss (B13xx)

Problem characterization as a subset of problem definition is performed through the Is and Is Not problem analysis (Table 2) and the function block diagram to understand the connection and interaction between the components of the equipment related to the issue. 
Proc. of the Third Intl. Conf. on Advances in Mechanical, Aeronautical and Production Techniques - MAPT 2015

Copyright (c) Institute of Research Engineers and Doctors, USA .All rights reserved.

ISBN: 978-1-63248-059-0 doi: 10.15224/ 978-1-63248-059-0-69

Table 2: Is-Is Not Analysis Of High Yield Loss (B13xx)

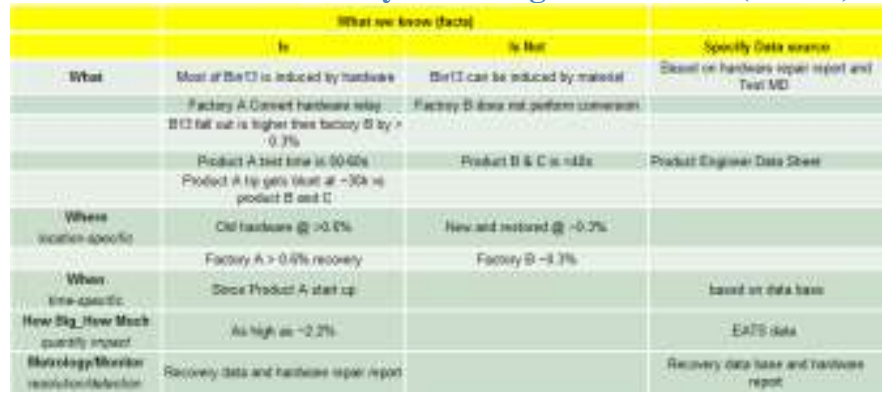

As shown in Figure 13, the team identifies the potential excessive and insufficient connection between the components for further experimentation through hypothesis development for tissue regeneration.

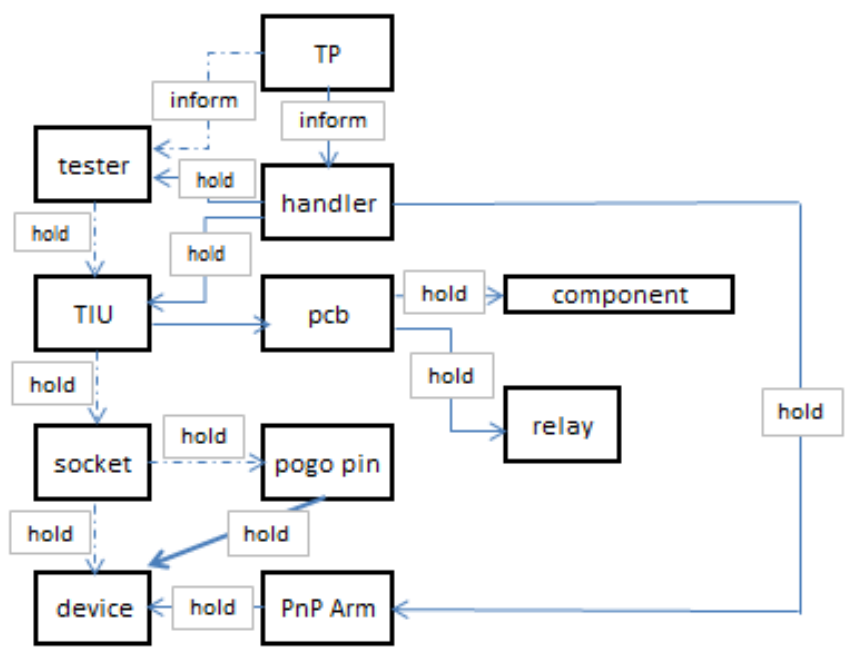

Figure 13: Function Block Model (B13xx)

In Stage 2, the model is developed based on the hypothesis and validated to identify the actual root cause. The validation indicates that the excessive power and test time degrade the life span of the pogo pin. Table 3 shows the development and validation of the high yield loss model for Bin13xx.

Table 3: High Yield Loss Model Development And Validation (B13xx)

\begin{tabular}{|c|c|c|c|}
\hline Component & Fonctio & Motel Development & Model Yaldation \\
\hline $\begin{array}{l}\text { Mandier Prif } \\
\text { |Mechanicail }\end{array}$ & Excensive & 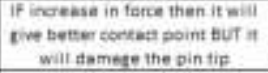 & $\begin{array}{c}\text { there s a hardatep to pretect } \\
\text { the pin vip Nat tewible and no } \\
\text { conristion }\end{array}$ \\
\hline $\begin{array}{l}\text { Pogro pin } \\
\text { (Thermsil }\end{array}$ & Excessive & $\begin{array}{l}\text { If the power applied lonser } \\
\text { Then will give o benter therme. } \\
\text { distribution out con impsot the } \\
\text { pin tip towards degradation }\end{array}$ & $\begin{array}{l}\text { With longer test tince, the powet } \\
\text { application wili be accessive } \\
\text { and slowly wil decrade the pin } \\
\text { tip }\end{array}$ \\
\hline $\begin{array}{l}\text { Pogofin } \\
\text { (Eletrirsil| }\end{array}$ & Excessive & 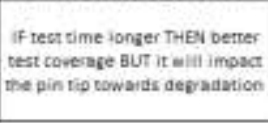 & 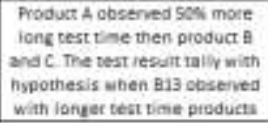 \\
\hline
\end{tabular}

A pogo pin is a device used in sockets to establish a connection between two printed circuit boards. The pogo pin is hard yet plated with a substance (gold) that ensures reliable contact (Figure 14).
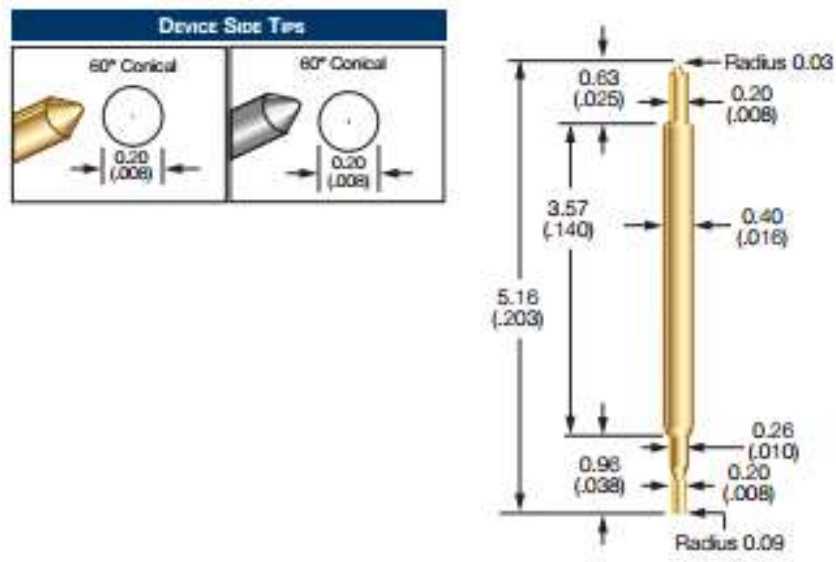

Figure 14: Pogo Pin

The findings show that the tip of the pogo pin becomes flat because of wear and tear (Figure 15).The model above signifies that the structure of the pogo pin changes with the combination of electrical power and thermal distribution under a given period.
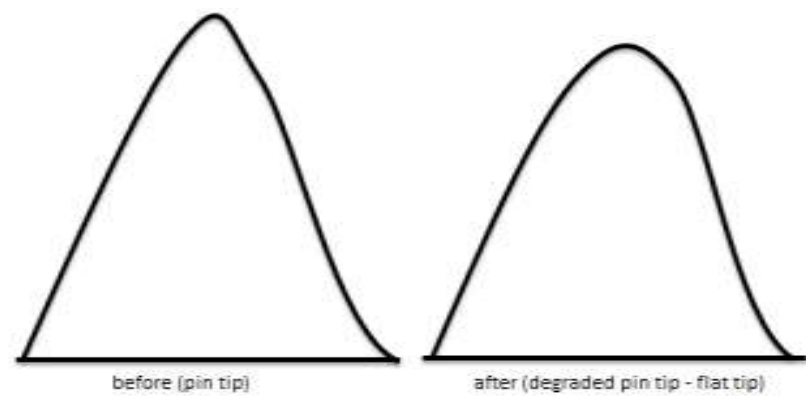

Figure 15: Tip Of Pogo Pin (Before And After)

Stage 3 involves the engineering contradiction and solution space, which has 40 potential inventive principles.

"IF power applied longer (increase in time) THEN gives a better thermal distribution (stability) BUT it will lead to pin tip degradation (durability)",

On the basis of the above IF, THEN, and BUT statement, we arrive at 39 contradiction parameters. Table 5.10 shows the 40 potential inventive principles. 
Table 4: High-Yield Loss Engineering Contradiction (B13xx)

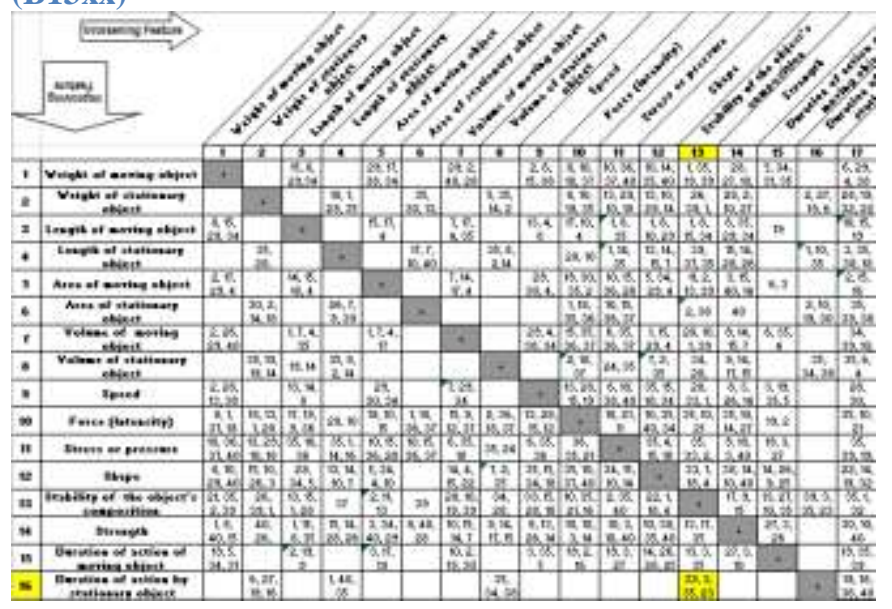

\section{Inert Atmosphere}

a. Use inert gases instead of usual ones.

b. Add neutral parts or additives to the object.

\section{Change of Phsical and Chemical Parameters}

a. Change the object's aggregate state.

b. Change concentration or composition of the object.

c. Change the degree of flexibility of the object.

d. Change the temperature of the object or environment.

Principle 39 of the periodic action proposes the addition of a neutral part or an inert additive to an object, and principle 35 of the parameter change proposes to change the object state/material. The technical team of the firm under study produced a proof of concept with a homogenous (single material) pogo pin to test pin tip degradation that occurs under the current setting. A homogenous pin made from customdeveloped solid precious metal alloy can be used without any additional plating process and features a robust structure. With this pin, the uptime is increased, and the lifespan of the pogo pin improves, resulting in a yield of up to $80 \%$. Figure 16 shows comparison between a new tip and its state after 400,000 cycles. The structure of the pin is clearly maintained and shows no sign of degradation.

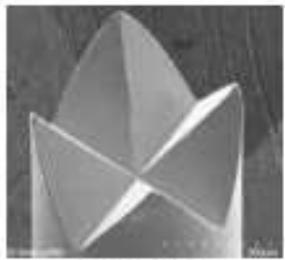

New Tip

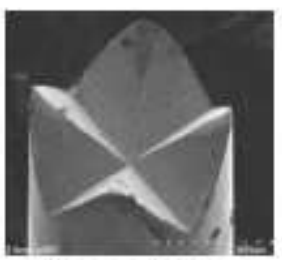

Clean and Sharp After $400 \mathrm{~K}$ Cycles
Figure 16: New Pogo Pin Tip Design

Chart 1 shows that the outcome of the new inventive pin brought down the yield loss trend from approximately $0.6 \%$ to $0.15 \%$. Upon a successful three-month data collection based on the proof of concept implementation, the team decided to apply the concept to all the affected hardware, thereby ensuring standardization among the hardware applications.

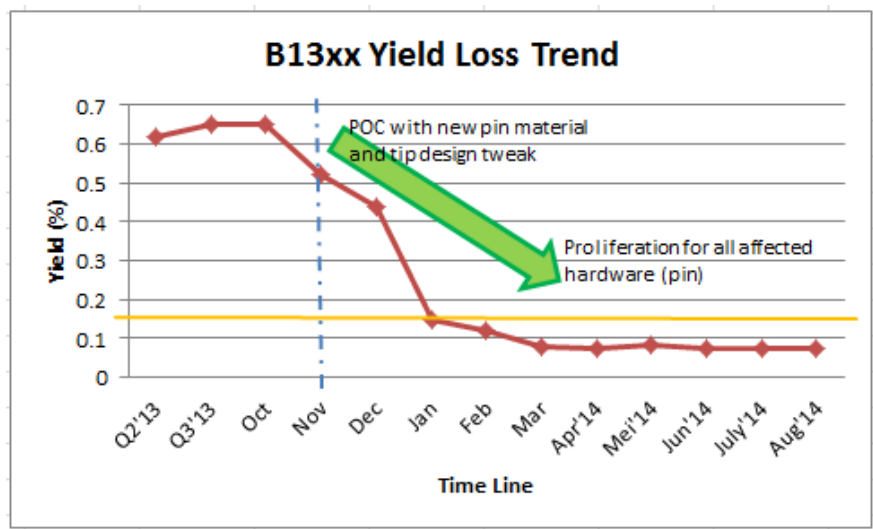

Chart 1: Yield-Loss Trend (before/after; B13xx)

MBSA has been validated with several case studies with different scopes of analysis. The case studies conducted in the validation stage vary in terms of system, process, and mechanism. Regardless of the number of systems involved in this analysis, each case study achieves the main objective of the MBSA and demonstrates its robustness from the flexibility and feasibility perspective.

The approaches with and without the MBSA are different. The case study without the MBSA is time consuming because the problem is contained based only on perception, in which case the problem is deemed controlled when it does not emerge within a short period. However, in the firm under study, the problem emerged after three months of implementation. The MBSA is used to explore the actual problem statement, as well as to have an in-depth problem segmentation and characterization. Table 5 shows the comparison between the approaches with and without the MBSA and the corresponding effects.

Table 5: Comparison Between Approaches With And Without The MBSA

\begin{tabular}{|c|c|}
\hline Withosit mess & With Musa \\
\hline NOPSM-trial -error, insight & New PSM - MBSA \\
\hline no model & every stage has its own model reference \\
\hline NO foel proaf solution - problem recocurance & Fool profl solution- no reocrurance \\
\hline $\begin{array}{l}\text { solutions are considered by comparison practical } \\
\text { System with and wathout success }\end{array}$ & $\begin{array}{l}\text { solutions are crested based on model diagram, } \\
\text { and iewentive principles }\end{array}$ \\
\hline no structured leads to long lead time & simplified $<2$ weeks \\
\hline knowiedge based to make a docision & data based decoisian making \\
\hline minimiged quality impect & Eliminate quality impese \\
\hline
\end{tabular}

note PSM - probiem solving nodel

Table 5 also concludes that the MBSA enables a detailed root cause analysis, which can derive a foolproof solution. The MBSA adopts a clustered pattern instead of a trial-and-error or insight pattern to facilitate solution derivation. The MBSA can further be extended to risk management in semiconductor manufacturing. The main focus is on improving and addressing the potential gaps in the FMEA. This focus would help ensure that the FMEA is equipped with a control solution for each potential issue. 
Most PSMs do not have an in-depth or structured model for the problem space. This deficiency results in a method that only contains the problem fora short period. As time passes, the problem is bound to occur again. Thus, any problem that is being solved must have a permanent corresponding solution to avoid its recurrence. The case study in this paper involves neither foolproof solutions nor a chronic issue without available solutions.

In summary, this pioneering MBSA research is based on observations of the manufacturing industry conducted through case studies. The MBSA is derived from observations of proposed business practices. The model integrates all processes, thereby providing a structured PSM for crossindustry explorations. The MBSA is an enhancement of the integration of various models and knowledge within the PSMs.

\section{CONCLUSION}

This research presents a structured and systematic approach for developing a PSM that can be effectively used to identify the real root causes of problems and develop foolproof solutions. This research uses the literature as basis to discover the concept for the new PSM and to develop, verify, and validate the developed model through case studies. The challenges lie in the development of the model to ensure its robustness and feasibility toward real-world issues with different mechanisms.

Over 80 publications on operational excellence and PSM-related papers are included in the literature review process. On the basis of this review, we conclude that developing a structured PSM that is feasible for manufacturing industries is more important than developing a theoretical advanced PSM. Considering the findings presented in Chapter 2 , we also conclude that the integration of the clustering model into the problem definition (problem statement, problem characterization, and problem segmentation) and model development/validation (Is-Is Not) results in the derivation of a good solution (through the TRIZ inventive principle).

The most significant attributes of the MBSA is the fundamental focus on the PSM, which involves problem definition using what-why analysis, problem segmentation, and problem characterization for in-depth problem analysis. The MBSA is comprehensively detailed at each stage for a strong fundamental understanding of the problem definition. Following the model development and validation stage, the focus shifts on the analysis of the major root cause of a problem through the recreation of the issue using hypothesis building. Through an engineering contradiction, detailed inventive solutions are listed for optimum solution prioritization. The model helps reduce the time spent on solving a problem and identifying the actual root cause of the problem. Through the case studies, we prove that the MBSA is applicable in highly complex equipment problems for it leads to the generation of control and foolproof solutions. This study also proves that the MBSA offers significant advantages to industry experts. The improved results of the MBSA leads to the rapid development of a solution, the analysis for which requires only a short period. In addition, industry experts can easily understand this model because of the structured procedure that facilitates the PSM.

The proposed MBSA is proved practical and applied successfully in the target industry of this research. The PSM has become an important element in solving chronic issues in the manufacturing industry. The MBSA can eliminate the conventional time-consuming practice of using the trial-anderror model. Meanwhile, the outcome of the MBSA is valuable for solving problems and avoiding their recurrence, sustaining equipment stability, and ensuring that no quality issues are repeated. In other words, the MBSA can also be a guide for further enhancing the effectiveness of industry experts in problem solving.

The verification and validation stage of the MBSA provides a clear goal for evaluating and determining the optimal PSM for the manufacturing industry. This verification and validation process proves the robustness of the MBSA in terms of feasibility, acceptability, and ease of use in the manufacturing industry.

In summary, the MBSA is proved to be capable of providing a structured and systematic way of solving a problem with corresponding criteria under different circumstances. The MBSA is also useful in coping with the business strategies formed by top management. The three key objectives of the research are clearly achieved. This model can also be effectively used to address any engineering problems and increase the competency level of technical experts. The organization that implements this model can easily solve problems in a timely manner, successfully mitigate prospective quality issues using the model, develop a training plan to address the identified needs, and deliver appropriate training for targeted skills. The ultimate aim is to maximize the problem-solving skills and effectiveness of the organization through the deployment of the MBSA to employees.

\section{ACKNOWLEDGMENT}

This paper was prepared with the aid of my full-time research and practice in Intel Technology Sdn. Bhd. I would like to thank my employer for funding the Doctor of Philosophy program fees and providing the necessary support and encouragement that allowed me to convert my research and work into academic writing. I would like to thank Dr. TS Yeoh for his guidance and patience in the internal review and approval process, which allowed this dissertation to be published without violating the company policies on intellectual property. I wish to express my sincere appreciation for my academic advisor, Assoc. Prof. Dr. Shahrul Kamaruddin, who patiently guided me in the completion of this dissertation and provided me with valuable feedback. I would also like to thank my second academic advisor, Dr. Ishak Abdul Azid, who reviewed my dissertation, provided important inputs, and offered motivation. I am especially grateful to my family for bearing with me as I completed my doctorate while engaging in full-time work. 
Proc. of the Third Intl. Conf. on Advances in Mechanical, Aeronautical and Production Techniques - MAPT 2015

Copyright (c) Institute of Research Engineers and Doctors, USA .All rights reserved. ISBN: 978-1-63248-059-0 doi: 10.15224/ 978-1-63248-059-0-69

\section{REFERENCES}

[1] Newell, A., \& Simon, , H. (1972). Human Problem Solving. Eaglewood Cliffs, NJ: Prentice Hall.

[2] Nutt, P. (1999). Suprising But True: Half The Decision In Organization Fail. Academy Of Management Executive, Vol 13 (4), pg75-90.

[3] Eastman, E. (2003). Fact Based Problem Identification Prcedes Problem Solving. JCSC Vol19.

[4] Polya, G. (1981). Mathematical Discovery. VOl 2. Princeton, NJ: Princeton University Press.

[5] Reed, S. (2002). Complex Cognition: The Psychology of Human Thought.

[6] Carson, J. (2007). A Problem With Problem Solving: Teaching Thinking Without Thinking Knowledge. The Mathematics Educator. Vol 17, No 2, pg 7 -14.

[7] Schooler, J., Ohlsson, S., \& Brooks, K. (1993). Thoughts beyond Words: When language Overshadows Insight. Journal of Experimental Psychology: General, 122, pg166-183

[8] Pearl, J. (1983). Knowledge versus search: A quantitative analysis using $A^{*}$. Artificial Intelligence:20, 1-13

[9] Anderson, D., Burnham, K., \& Thompson, W. (2000). Null Hypothesis Testing: Problems, Prevalence, And An Alternative. Journal of Wildlife Management 64 (4), pg912 - 923.

[10] Thorpe, H., Hawkins, K., Brown, J., \& Brown, S. (2000). Minimization: Reducing Predictability for Multi-Centre Trials Whilst Retaining Balance Within Centre. Journal of The Royal Statistical Society Series, pg359-370.

[11] Paletz, S., \& Peng, K. (2009). Problem Finding and Contradiction: examining the Relationship Between Naive Dialectical Thinking, ethnicity, and Creativity. Creativity Research Journal, 21 (2-3), pg139-151.

[12] Howard-Jones, P., \& Martin, R. (2002). The Effect of Questioning on Concept Learning Within a Hypertext System. Journal of Computer Assisted Learning. Vol 18 (1), Pg10-20.

[13] Bridget, R.-R., \& Gregory L., R.-R. (2011). CognitivePsychology 3rd Edition. Pearson.

[14] Weiten, D. \& McCann, D. (2007). Psychology: Theme and Variations ( $1^{\text {st }}$ Cam. Ed.) Toronto: Thomson \& Nelson

[15] Barbati, M., Bruno, G., \& Genovese, A. (2012). Applications of Agent Based Models for Optimization Problems: ALiterature Review. Expert Systems With Applications,39/5, pg6020- 6028.

[16] Kim, B., \& Kim, H. (2014). Sensitivity of earned Value Schedule Forecasting to S-Curve Patterns. Journal of Construction Engineering and Management Vol. 140 Issue 7.

[17] Lapidot, I., \& Conley, D. (2011). EvolutionPredictability, Lamarck, Altshuller, Darwin and Chaos. ETRIA TRIZ Future Conference. Dublin:Innomationcorp.

[18] Yin, R. (2003). Case Study Research: Design and Methods. SAGE Publication Inc.

[19] Whetten, D. (1989). What Constututes a Theoritical Contribution. Academy of Management Review. Vol14 (4), pg490-495.

[20] Runeson, \& Host, M. (2009). Guideline For Conducting and Reporting Case Study Research in SoftwareEngineering. Empirical Software Engineering. Vol 14(2), pg131-164.

[21] Meredith, J. (1998). Building Operations Management Theory Through Case and Field Research. Journal of Operations Management Vol 16, 441-454.

[22] Becker, H. (1970). Sociological Work: Method and Substance. Chicago IL: Aldine.

[23] Charles Kettering, F. (1947). Engineering Horizons. Journal of The American Society for Naval Engineering. Vol 59(2), pg277290.
[24] Durlak, J., \& DuPre, E. (2008). Implementation Matters: A review of Research on The Influence of Implementation on Program Outcomes And The Factors Affecting Implementation. AM J Community Psychology. Vol 41(3-4), pg327-350.

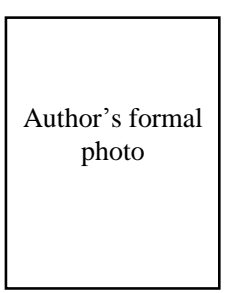

Nagappan Annamalai was born from Perak, Malaysia in 1977. He has a Beng (Hons) majored in Electrical and Electronics Engineering from University Northumbria, Newcastle, UK. Currently he is in midst of completing research Ph.D majored in Mechanical Engineering with Universiti Sains Malaysia. He also works for Intel Cooperation based in Penang, Malaysia as a Manufacturing Technologist. His field of interest to explore in problem solving methodology, process control system, and high precision maintenance.

Shahrul Kamaruddin born in Penang, Malaysia in 1972. He received the B.Eng.(Hons) degree from the University of Strathclyde, Glasgow, Scotland in 1996, the M.Sc. degree from University of Birmingham, U.K., in 1998, and the $\mathrm{PhD}$ from University of Birmingham, in 2003. Currently he is an Associate Professor with the School Mechanical Engineering (under the manufacturing engineering with management program), Universiti Sains Malaysia. He has various past experiences with manufacturing industries from heavy to electronics industries especially in the field of industrial engineering, manufacturing processes and product design. He has more 140 publications in reputed international and national journals/conferences. His current research interests include simulation and modeling of manufacturing systems, production planning and control, maintenance engineering and management, and manufacturing process related to plastic material.

Yeoh Teong San was born in Malacca in 1960. He has a Ph.D in Applied Physics from USM. He was formerly working in Intel Corporation in Malaysia as a Principal Engineer for the past 25 years. Currently, he is working on training and consultancy in problem solving and innovation. His passion and focus is on training, proliferation, and application of TRIZ (Theory of Inventive Problem Solving) into universities, colleges, schools, and industries; extending across various countries including China, Costa Rica, India, Malaysia, USA, Philippines, and Vietnam. He is also one of the founders of the Malaysia TRIZ Innovation Association (MyTRIZ) 